\title{
Further results on entanglement detection and quantification from the correlation matrix criterion
}

\author{
Julio I de Vicente \\ Departamento de Matemáticas, Universidad Carlos III de Madrid, Avda. de la \\ Universidad 30, 28911 Leganés, Madrid, Spain \\ E-mail: jdvicent@math.uc3m.es
}

\begin{abstract}
The correlation matrix $(\mathrm{CM})$ criterion is a recently derived powerful sufficient condition for the presence of entanglement in bipartite quantum states of arbitrary dimensions. It has been shown that it can be stronger than the positive partial transpose (PPT) criterion, as well as the computable cross norm or realignment (CCNR) criterion in different situations. However, it remained as an open question whether there existed sets of states for which the CM criterion could be stronger than both criteria simultaneously. Here, we give an affirmative answer to this question by providing examples of entangled states that scape detection by both the PPT and CCNR criteria whose entanglement is revealed by the CM condition. We also show that the CM can be used to measure the entanglement of pure states and obtain lower bounds for the entanglement measure known as tangle for general (mixed) states.
\end{abstract}

PACS numbers: 03.67.Mn, 03.65.Ud

Submitted to: J. Phys. A: Math. Gen. 


\section{Introduction}

Entanglement in composite systems is a characteristic feature of quantum mechanics which plays a key role in the foundations of the theory. Moreover, it is a fundamental resource in many of the applications of quantum information theory [1]. Therefore, the characterization of entangled states (the so-called separability problem) is of great interest, since a complete understanding of their structure would lead to a better understanding of quantum theory and it would also clarify which states are useful for quantum information tasks (e.g., in an experimental context). However, and despite many efforts in the last decade, the separability problem remains unsolved. In fact, it has been proved to be NP-hard [2], although several authors have devised nontrivial algorithms for it (see [3] for a survey). Nevertheless, there exist a great variety of analytical approaches to the problem which, besides their entanglement detection capability, give as a by-product a better insight in the structure of entangled states from the theoretical point of view. Historically, Bell inequalities were the first tool for the recognition of entanglement; however, it is well-known for some time that the violation of a Bell inequality is only a sufficient condition for entanglement and not a necessary one, and that there are in fact many entangled states that satisfy them (see, e.g., [4]). The most remarkable sufficient condition for the detection of entanglement is given by the Peres-Horodecki or positive partial transpose (PPT) criterion [5]. This condition is so strong that it characterizes entanglement for $2 \times 2$ and $2 \times 3$ systems [6], but not otherwise. Much subsequent work has been devoted to finding different sufficient conditions for the presence of entanglement that could complement the strong Peres-Horodecki criterion by detecting PPT entanglement (i.e., entangled states which are not detected by this criterion). The most remarkable one is the computable cross norm [7] or realignment [8] (CCNR) criterion, which exhibits a powerful PPT entanglement detection capability. There are also other important criteria with this property, which, however, lack the operational character (i.e., ease of use) of the aforementioned ones because they rely on expectation values of observables which have to be chosen appropriately for the state in question. This is the case of conditions based on entanglement witnesses (see e.g. [6, 9]) or uncertainty relations [10].

In a recent paper [11, a new easily computable operational sufficient condition for entanglement in bipartite quantum systems of arbitrary dimensions $M \times N$ has been obtained by studying separability from the point of view of the Bloch representation of density matrices. We will refer to it as correlation matrix (CM) criterion. It was proved that it is able to recognize PPT entanglement when $M=N$, and that, although strictly weaker than the CCNR criterion when $M=N$ (i.e., the CCNR criterion detects all entangled states recognized by the CM criterion in this situation and not conversely), it can be stronger when $M \neq N$. Therefore, it remained as an open question whether the CM criterion could be stronger than both the PPT and CCNR criteria for certain states. We will show in this paper that this is indeed the case by providing examples of PPT entangled states not detected by the CCNR criterion whose entanglement is revealed by 
the CM criterion. This result further confirms the interest of the CM criterion, showing that it actually complements the PPT and CCNR criteria improving our ability to detect entanglement. We will also consider a very recently derived criterion [12, which is strictly stronger than the CCNR.

In the last part of this paper we will consider the possibility of quantifying entanglement through the CM. Besides the characterization of entanglement, the derivation of good entanglement measures is a fundamental problem in quantum information theory since they provide a way to quantify how useful an entangled state is for a certain quantum information process. Several measures of this type exist (see, e.g., the recent survey [13]), but, in general, their explicit computation is a very hard task, even numerically. Therefore, it is interesting to find good bounds for them (see [13, 14] and references therein). It is widely believed that a stronger violation of a separability condition indicates a bigger amount of entanglement, and, in fact it has been shown that they can be used to place lower bounds on different entanglement measures [15, 16, 17] (see also [18] for bounds obtained by considering two separability conditions simultaneously). In particular, the measure known as concurrence can be bounded from below using the PPT and CCNR criteria [15], and the CM criterion [17]. These results can be used to obtain lower bounds for its close cousin the tangle. However, we will prove here that the CM can be used to obtain different lower bounds for this measure, which are particularly sharp for states of high purity. This follows from the fact that the CM provides the actual value of both tangle and concurrence for pure states as we will also show. Finally, as an application, we will use this new bound to prove a recently conjectured result relating concurrence and the so-called Minkowskiannorm-based (MNB) entanglement measure for two-qubit systems $(M=N=2)$ [19, 20].

\section{Separability, Bloch representation and CM criterion}

Consider a bipartite quantum system composed of subsystems $A$ and $B$, of $M$ and $N$ levels respectively. Then, its quantum state is characterized by the density operator $\rho \in \mathcal{B}\left(H_{A} \otimes H_{B}\right)$, where $H_{A} \simeq \mathbb{C}^{M}$ and $H_{B} \simeq \mathbb{C}^{N}$ denote the Hilbert spaces of the subsystems and $\mathcal{B}(H)$ stands for the real vector space of Hermitian operators acting on $H$ with the standard Hilbert-Schmidt inner product $\langle\rho, \tau\rangle_{H S}=\operatorname{Tr}\left(\rho^{\dagger} \tau\right)$. The state is said to be separable (entangled) if it can (cannot) be written as a convex combination of product states [4], i. e.

$$
\rho=\sum_{i} p_{i} \rho_{i}^{A} \otimes \rho_{i}^{B}
$$

where $0 \leq p_{i} \leq 1, \sum_{i} p_{i}=1$, and $\rho_{i}^{A}\left(\rho_{i}^{B}\right)$ denotes a pure state density matrix acting on $H_{A}\left(H_{B}\right)$. Given that it is in general very hard to verify if a decomposition according to the definition of separability (11) exists for a given state, the separability problem consists in finding computable mathematical conditions which provide a practical way to check whether a given state is entangled or not. The PPT and CCNR criteria can be formulated in several ways. They can be easily applied by considering certain 
rearrangements of the matrix elements of $\rho$ [21]. For instance, if we expand with respect to the canonical product basis

$$
\rho=\sum_{i j k l} \rho_{i j, k l}|i j\rangle\langle k l|
$$

the PPT criterion states that for separable states the partial transpose $T_{A}(\rho)$, i.e.

$$
T_{A}(\rho)_{i j, k l}=\rho_{k j, i l},
$$

still represents a state and it is, therefore, positive semidefinite, which means that $\left\|T_{A}(\rho)\right\|_{t r}=1$, where here and throughout the paper $\|\cdot\|_{t r}$ stands for the trace or Ky Fan norm (i.e. the sum of the singular values). Similarly, the CCNR criterion affirms that the realignment operation $R(\rho)$,

$$
R(\rho)_{i j, k l}=\rho_{i k, j l}
$$

is such that $\|R(\rho)\|_{t r} \leq 1$ for all separable states. Hence, $\left\|T_{A}(\rho)\right\|_{t r},\|R(\rho)\|_{t r}>1$ is a signature of entanglement.

To introduce the CM criterion we first say a few words about the Bloch representation of density matrices [22, 23], which is an expansion of $\rho$ in terms of the orthogonal basis of $\mathcal{B}(H)$ given by the identity $I$ and the traceless Hermitian generators of the group $S U(\operatorname{dim} H)\left\{\lambda_{i}\right\}$,

$$
\rho=\frac{1}{\operatorname{dim} H}\left(I+\sum_{i=1}^{(\operatorname{dim} H)^{2}-1} r_{i} \lambda_{i}\right)
$$

The generators fulfill the following orthogonality relations (the normalization is chosen by standard usage),

$$
\left\langle\lambda_{i}, I\right\rangle_{H S}=\operatorname{Tr}\left(\lambda_{i}\right)=0, \quad\left\langle\lambda_{i}, \lambda_{j}\right\rangle_{H S}=\operatorname{Tr}\left(\lambda_{i} \lambda_{j}\right)=2 \delta_{i j}
$$

and they can be easily constructed from any orthonormal basis in $H$ [23]. The coefficients $\left\{r_{i}\right\}$ which completely characterize $\rho$ form the coherence or Bloch vector $\mathbf{r} \in \mathbb{R}^{(\operatorname{dim} H)^{2}-1}$. The orthogonality of the basis implies that this vector can be easily determined (theoretically and experimentally) from the expectation values of the observables $\left\{\lambda_{i}\right\}$

$$
r_{i}=\frac{\operatorname{dim} H}{2}\left\langle\rho, \lambda_{i}\right\rangle_{H S}=\frac{\operatorname{dim} H}{2} \operatorname{Tr}\left(\rho \lambda_{i}\right)=\frac{\operatorname{dim} H}{2}\left\langle\lambda_{i}\right\rangle_{\rho} .
$$

In the case of $M \times N$ bipartite quantum systems ( $M \leq N$ is assumed without loss of generality throughout the paper), the Bloch representation (also known as Fano form [24]) can be written as

$\rho=\frac{1}{M N}\left(I_{M} \otimes I_{N}+\sum_{i} r_{i} \lambda_{i}^{A} \otimes I_{N}+\sum_{j} s_{j} I_{M} \otimes \lambda_{j}^{B}+\sum_{i, j} t_{i j} \lambda_{i}^{A} \otimes \lambda_{j}^{B}\right)$,

where $\left\{\lambda_{i}^{A}\right\}_{i=1}^{M^{2}-1}$ and $\left\{\lambda_{i}^{B}\right\}_{i=1}^{N^{2}-1}$ denote the generators of $S U(M)$ and $S U(N)$. This representation has to kind of parameters: $\left\{r_{i}\right\}=M / 2\left\{\left\langle\lambda_{i}^{A} \otimes I_{N}\right\rangle_{\rho}\right\}$ and $\left\{s_{i}\right\}=$ $N / 2\left\{\left\langle I_{M} \otimes \lambda_{j}^{B}\right\rangle_{\rho}\right\}$, which are local since they are the Bloch parameters of the reductions $\left(\rho_{A}=\operatorname{Tr}_{B} \rho=1 / M\left(I+\sum_{i} r_{i} \lambda_{i}^{A}\right), \rho_{B}=\operatorname{Tr}_{A} \rho=1 / N\left(I+\sum_{i} s_{i} \lambda_{i}^{B}\right)\right)$; and $\left\{t_{i j}\right\}=$ 
$M N / 4\left\{\left\langle\lambda_{i}^{A} \otimes \lambda_{j}^{B}\right\rangle_{\rho}\right\}$, which are responsible for the possible correlations between the subsystems and form the CM $T \in \mathbb{R}^{\left(M^{2}-1\right) \times\left(N^{2}-1\right)}$. The CM criterion states that

$$
\|T\|_{t r} \leq \sqrt{\frac{M N(M-1)(N-1)}{4}}
$$

must hold for all separable states [11. This implies that there is an upper bound to the "amount" of correlations contained in this kind of states, a higher degree of correlations only being achievable through entanglement.

\section{Entangled states detected by the CM criterion but not by the PPT and CCNR criteria}

In [11] it is proved that the CCNR criterion is stronger than the CM criterion when $M=N$. However, in the case of states with maximally mixed subsystems (i.e., $\mathbf{r}=\mathbf{s}=0)$ it is shown that the CM criterion is strictly stronger than the CCNR criterion when $M \neq N$, being equivalent when $M=N$. Several examples of $M=N \mathrm{PPT}$ entangled states detected by condition (9) are also provided. However, since the CCNR condition is stronger in this case, the entanglement of all these states is already revealed by this criterion. Therefore, to find examples of entangled states just detected by the CM criterion we have to restrict ourselves to the $M \neq N$ case. Unfortunately, although many examples of PPT entangled states are known when $M=N$ (see, e.g., [25, 26, 27]), the situation in the asymmetric case is not as rich. In [25] a set of PPT entangled states in $2 \times 4$ dimensions is provided (see the paper for their explicit form). However, it can be readily checked that both the CCNR and CM criteria are unable to identify these states as entangled as well. In [26], an entangled PPT state with subsystems of different dimensions $(M, N \geq 3)$ is constructed from the unextendible product basis GenTiles2 (its explicit form is given below). However, while the CCNR criterion detects entanglement for this state when $M=3,4 \leq N \leq 8$ and when $M=4, N=5$, the $\mathrm{CM}$ criterion only recognizes entanglement in the $3 \times 4$ case. So, unfortunately for our purpose, the CCNR criterion seems stronger for these states. The entanglement properties of rotationally invariant states has been thoroughly studied in recent literature. In particular, the set of PPT rotationally invariant states has been determined in $3 \times N$ [28] and $4 \times N$ [29] systems, it has been shown that the PeresHorodecki criterion does not characterize entanglement in this scenario and many PPT entangled states have been identified. Although this situation may seem promising to our purpose since rotationally invariant states have maximally disordered subsystems and, therefore, the CM criterion is guaranteed to improve on the CCNR criterion, our numerical explorations indicate that the PPT criterion is stronger than the CM criterion for these states.

The above states are, to our knowledge, the only examples available in the literature of PPT entanglement with subsystems of different dimensions. Therefore, to achieve our goal we have to either construct new examples or to consider PPT-preserving operations on the previous states which transform them to different states in which the CM criterion 
is stronger than the CCNR, such as the $\mathbf{r}=\mathbf{s}=0$ case. It turns out that this transformations are very well studied. In [30] (see also [31]) it is shown that every full-rank state can be transformed under the action of local filtering operations (also known as stochastic local operations assisted by classical communication SLOCC) into a state with maximally mixed subsystems which is called (filter) normal form (FNF). This form is unique up to local unitary transformations. Mathematically, the filtering operations are represented by invertible matrices $F_{A}, F_{B}$ (without loss of generality they can be chosen of determinant equal to one) which transform $\rho$ into $\tilde{\rho}$ according to

$$
\tilde{\rho}=\frac{\left(F_{A} \otimes F_{B}\right) \rho\left(F_{A} \otimes F_{B}\right)^{\dagger}}{\operatorname{Tr}\left(F_{A} \otimes F_{B}\right) \rho\left(F_{A} \otimes F_{B}\right)^{\dagger}} .
$$

There exist several constructive algorithms which provide the matrices $F_{A}, F_{B}$ needed in order to take a given state into its normal form [30, 31] (see also [12]). In particular, there is a matlab code available in [32]. Although the existence of the FNF is guaranteed only for full-rank density matrices, rank-deficient states can be brought to a state whose reductions are arbitrarily close to the maximally mixed state using the aforementioned algorithms. The transformation given by (10) preserves the separability or entanglement of a given state as well as the PPT property, so the CM criterion will be stronger than the PPT and CCNR criteria for states $\tilde{\rho}$ in the FNF obtained from a PPT entangled state. The FNF was first considered in the context of the separability problem in [12]: since these SLOCC transformations that wash out all the local information maximize the entanglement content of a state in a certain sense [30], the entanglement detection capability of separability conditions is greatly improved in the FNF. However, here we will just consider it as a way to construct a state with some desired properties.

We start by considering the entangled PPT state $\rho_{G T 2}$ constructed from the unextendible product basis GenTiles2 [27, 33] in $M \times N$ dimensions such that $M \geq 3$, $N>3$ and $M \leq N$,

$\rho_{G T 2}=\frac{1}{2 M-1}\left(I_{M N}-|F\rangle\left\langle F\left|-\sum_{j=0}^{M-1}\right| S_{j}\right\rangle\left\langle S_{j}\left|-\sum_{j=0}^{M-1} \sum_{k=1}^{N-3}\right| L_{j k}\right\rangle\left\langle L_{j k}\right|\right)$,

where

$$
\begin{aligned}
|F\rangle & =\frac{1}{\sqrt{M N}} \sum_{i=0}^{M-1} \sum_{j=0}^{N-1}|i j\rangle, \\
\left|S_{j}\right\rangle & =\frac{1}{\sqrt{2}}(|j\rangle-|j+1 \bmod M\rangle)|j\rangle, \\
\left|L_{j k}\right\rangle & =\frac{1}{\sqrt{N-2}}|j\rangle\left(\sum_{l=0}^{M-3} \exp \left(\mathrm{i} \frac{2 \pi l k}{N-2}\right)|l+j+1 \bmod M\rangle\right. \\
& \left.+\sum_{l=M-2}^{N-3} \exp \left(\mathrm{i} \frac{2 \pi l k}{N-2}\right)|l+2\rangle\right) .
\end{aligned}
$$

For simplicity we restrict ourselves to the lowest possible dimensions $M=3$ and $N=4$. In this case $\left\|T_{\rho_{G T 2}} \mid\right\|_{t r}=4.3428$ and $\left\|R\left(\rho_{G T 2}\right)\right\|_{t r}=1.0315$ and, therefore, as said before, 
both the $\mathrm{CM}$ and $\mathrm{CCNR}$ criteria detect $\rho_{G T 2}$ as entangled. It can be seen by considering any of the algorithms mentioned above that the filters

$$
\begin{aligned}
& F_{A}=\left(\begin{array}{rrr}
-0.2586-0.4251 \mathrm{i} & -0.2586-0.4251 \mathrm{i} & -0.2586-0.4251 \mathrm{i} \\
0.3421-0.3842 \mathrm{i} & 0.4402+0.2817 \mathrm{i} & -0.7824+0.1025 \mathrm{i} \\
0.2784-0.6568 \mathrm{i} & -0.5774+0.4086 \mathrm{i} & 0.2990+0.2482 \mathrm{i}
\end{array}\right), \\
& F_{B}=\left(\begin{array}{rrrr}
-0.3118-0.3092 \mathrm{i} & -0.3118-0.3092 \mathrm{i} & -0.3118-0.3092 \mathrm{i} & -0.3118-0.3092 \mathrm{i} \\
0.5499-0.2805 \mathrm{i} & 0.6414-0.0813 \mathrm{i} & -0.3307+0.0334 \mathrm{i} & -0.4303+0.1642 \mathrm{i} \\
-0.3932-0.1066 \mathrm{i} & 0.3198-0.3909 \mathrm{i} & -0.0427-0.7619 \mathrm{i} & 0.0580+0.6297 \mathrm{i} \\
0.5358+0.3605 \mathrm{i} & 0.1113-0.5279 \mathrm{i} & 0.5169-0.0640 \mathrm{i} & -0.5820+0.1157 \mathrm{i}
\end{array}\right),
\end{aligned}
$$

transform $\rho_{G T 2}$ onto its normal form $\tilde{\rho}_{G T 2}$. Now, we readily find that $\left\|T_{\tilde{\rho}_{G T 2}}\right\|_{t_{r}}=4.5751$ and $\left\|R\left(\tilde{\rho}_{G T 2}\right)\right\|_{t r}=1.0512$, and again both the CM and CCNR criteria reveal the entanglement of this PPT state. However, the CM criterion is now stronger and, therefore, more robust against noise. So if we consider the previous state mixed with white noise, i.e.

$$
\rho(p)=p \tilde{\rho}_{G T 2}+(1-p) \frac{I_{12}}{12},
$$

we find that the $\mathrm{CM}$ criterion detects entanglement in $\rho(p)$ whenever $p \geq 0.9274$ while the CCNR criterion recognizes entanglement when $p \geq 0.9330$. Notice that by construction the PPT criterion is unable to find entanglement in $\rho(p)$. Thus, this example shows that the CM criterion can detect states which are neither detected by the CCNR criterion nor by the PPT criterion. Moreover, the recently powerful criterion based on covariance matrices derived in [12] (which is strictly stronger than the CCNR) detects entanglement in this state when $p \geq 0.9290$ and it is, therefore, also weaker than the CM criterion for these states, as was expected, since in the above mentioned paper it is shown that the new criterion is stronger than the $\mathrm{CM}$ criterion when $M \ll N$ but otherwise weaker in the case of states with maximally disordered subsystems. Several other examples of entangled states detected by the CM criterion but not by the PPT and CCNR criteria can be found considering the FNF of $\rho_{G T 2}$ for other values of $M$ and $N$. However, it is worth pointing out, that the FNF of the $2 \times 4$ states of [25] is still undetected by the CM and CCNR criteria and that, in fact, $\left\|T_{\tilde{\rho}}\right\|_{t r}<\left\|T_{\rho}\right\|_{t r}$ and $\|R(\tilde{\rho})\|_{t r}<\|R(\rho)\|_{t r}$ for many of these states.

\section{Tangle and the CM}

The entanglement of formation [34] is the only measure of entanglement for which an analytical expression is available for arbitrary systems of particular dimensions. It was found in 35 for the case of two-qubit systems. In this case the entanglement of formation is a monotonically increasing function of a quantity called concurrence $\mathrm{C}$, so $\mathrm{C}$ is taken as a measure of entanglement in its own right. Furthermore, it has been successfully generalized to arbitrary dimensional bipartite quantum states [36, 37] (see 
also [38]). For a pure state $\psi$, it is given by [36]

$$
C(\psi)=\sqrt{2\left(1-\operatorname{Tr} \rho_{A}^{2}\right)} .
$$

Notice that $0 \leq C(\psi) \leq \sqrt{2(M-1) / M}$, the lower bound being attained by product states and the upper bound by maximally entangled states. The definition is extended to general mixed states $\rho$ by the convex roof (the minimum average value of the pure-state measure over all possible ensemble realizations of $\rho$ ) [37],

$$
C(\rho)=\min _{\left\{p_{i},\left|\psi_{i}\right\rangle\right\}}\left\{\sum_{i} p_{i} C\left(\psi_{i}\right): \rho=\sum_{i} p_{i}\left|\psi_{i}\right\rangle\left\langle\psi_{i}\right|\right\} .
$$

Consequently, $C(\rho)=0$ if, and only if, $\rho$ is a separable state. It can be more convenient to remove the square root in (14) and consider the measure $\tau(\psi)=C^{2}(\psi)$, which is then extended to mixed states by the convex roof

$$
\tau(\rho)=\min _{\left\{p_{i},\left|\psi_{i}\right\rangle\right\}}\left\{\sum_{i} p_{i} C^{2}\left(\psi_{i}\right): \rho=\sum_{i} p_{i}\left|\psi_{i}\right\rangle\left\langle\psi_{i}\right|\right\} .
$$

The measure $\tau$ is known as tangle. Notice that, although equal to the squared concurrence for pure states, for general states it holds that $\tau(\rho) \geq C^{2}(\rho)$; nevertheless, it can be shown that $\tau(\rho)=C^{2}(\rho)$ in the case of two-qubit states (see [39]). Some authors have found the tangle a more natural measure than the concurrence because a closed formula can be derived for it for rank-2 density operators [39] and because, contrary to the concurrence, its behaviour is analogous to the entanglement of formation for isotropic states [37.

Due to the convex roof construction these measures are very hard to compute in the case of mixed states, so, as mentioned in Sec. I, good bounds for their estimation are desirable. In particular, lower bounds are preferable because upper bounds can be obtained considering any ensemble decomposition of the state. It seems natural to think that $\left\|T_{A}(\rho)\right\|_{t r}$ and $\|R(\rho)\|_{t r}$ provide an estimate of the entanglement content of $\rho$ since the greater than 1 they are, the further the state is to separability in a certain sense. In fact, it has been proved that [15]

$$
C(\rho) \geq \sqrt{\frac{2}{M(M-1)}}\left[\max \left(\left\|T_{A}(\rho)\right\|_{t r},\|R(\rho)\|_{t r}\right)-1\right],
$$

which provides a powerful lower bound to estimate the concurrence from these separability conditions. It has been shown in [17] that the CM can be used analogously since

$$
C(\rho) \geq \sqrt{\frac{8}{M^{3} N^{2}(M-1)}}\left(\|T\|_{t r}-\sqrt{\frac{M N(M-1)(N-1)}{4}}\right) .
$$

The bound given by (17) is generally tighter than that of (18) (see [17]). However, using the results of Sec. II we can provide examples of the contrary (which lacked in [17]). For instance, while (17) tells us that $C\left(\tilde{\rho}_{G T 2}\right) \geq 0.0296$, we have that $C\left(\tilde{\rho}_{G T 2}\right) \geq 0.0320$ using (18). The above formulas can also be used for the tangle recalling that $\tau(\rho) \geq C^{2}(\rho)$. 
However, here we will derive a different lower bound which is exclusively designed for the tangle which is better than Eqs. (17)-(18) in certain situations.

The matrix $T$ contains the information about the correlations between the subsystems and the CM criterion bounds the amount of correlations in a separable state using the trace norm to quantify them. However, this criterion can be stated using any matrix norm since the proof only relies on the triangle inequality [11]. As we shall discuss in more detail below, the choice of the trace norm is convenient because it provides the strongest separability condition; nevertheless, different choices can be more adequate if we are interested in the quantification of entanglement. This is the underlying idea for the new bound on the tangle to be derived in this Section. We first show that $\tau$ and $C$ are closely related to the CM for pure states and, moreover, that they can be evaluated by considering a particular norm of the CM.

\subsection{Pure states}

The concurrence and tangle of a pure state given by (14) can be easily written in terms of the parameters of the Bloch representation. Recall that the reduced density matrix of of an arbitrary state $\rho$ with Bloch representation (8) is $\rho_{A}=1 / M\left(I+\sum_{i} r_{i} \lambda_{i}^{A}\right)$. Thus, using (6), it can be seen that

$$
\operatorname{Tr}\left(\rho_{A}^{2}\right)=\frac{M+2\|\mathbf{r}\|_{2}^{2}}{M^{2}}
$$

where $\|\cdot\|_{2}$ is the Euclidean norm. Hence,

$$
\tau(\psi)=C^{2}(\psi)=\frac{2\left(M^{2}-M-2\|\mathbf{r}\|_{2}^{2}\right)}{M^{2}} .
$$

In the case of pure states $\mathbf{r}, \mathbf{s}$ and $T$ are related in a determined way. For these states the reductions $\rho_{A}$ and $\rho_{B}$ have the same eigenvalues and, therefore, $\operatorname{Tr}\left(\rho_{A}^{2}\right)=\operatorname{Tr}\left(\rho_{B}^{2}\right)$. So, recalling (19), this implies that

$$
\frac{M+2\|\mathbf{r}\|_{2}^{2}}{M^{2}}=\frac{N+2\|\mathbf{s}\|_{2}^{2}}{N^{2}}
$$

Furthermore, pure states satisfy $\operatorname{Tr}\left(\rho^{2}\right)=1$. Using again ([6) and some straightforward algebra, we readily see that this means that

$$
N\|\mathbf{r}\|_{2}^{2}+M\|\mathbf{s}\|_{2}^{2}+2\|T\|_{H S}^{2}=\frac{M N(M N-1)}{2},
$$

where $\|\cdot\|_{H S}$ is the Frobenius or Hilbert-Schmidt norm, that is, the norm induced by the Hilbert-Schmidt inner product, i.e.

$$
\|T\|_{H S}=\sqrt{\operatorname{Tr}\left(T^{\dagger} T\right)}=\sqrt{\sum_{i j}\left|t_{i j}\right|^{2}} .
$$

Eqs. (21) and (22) imply that the value of one of the parameters $\left\{\|\mathbf{r}\|_{2},\|\mathbf{s}\|_{2},\|T\|_{H S}\right\}$ uniquely determines the others in the case of pure states. Thus, inserting (21) in (22) we arrive at

$$
\|\mathbf{r}\|_{2}^{2}=\frac{M}{M+N}\left(\frac{N\left(M^{2}-1\right)}{2}-\frac{2}{N}\|T\|_{H S}^{2}\right) .
$$


Now, this last equation together with (20) lets us write the concurrence and tangle of an arbitrary pure bipartite state in terms of the CM,

$$
\tau(\psi)=C^{2}(\psi)=\frac{8}{M N(M+N)}\left(\|T\|_{H S}^{2}-\frac{M N(M-1)(N-1)}{4}\right) .
$$

In this way we see that for pure states, the CM not only characterizes entanglement but also enables to rigorously quantify it, since concurrence and tangle are functions of $\|T\|_{H S}$.

\subsection{Mixed states}

In the case of mixed states $\|T\|_{H S}$ cannot be used to express the tangle or concurrence in closed form as in (25). However, if we restrict ourselves to the tangle, it is possible to derive a lower bound for this measure similar to (18) using ||$T||_{H S}$. Let $\sum_{n} p_{n}\left|\psi_{n}\right\rangle\left\langle\psi_{n}\right|$ be the decomposition of $\rho$ for which the minimum in (16) is attained. Then, we have that

$$
\begin{aligned}
\tau(\rho) & =\sum_{n} p_{n} \tau\left(\psi_{n}\right) \\
& =\frac{8}{M N(M+N)}\left(\sum_{n} p_{n}\left\|T_{\psi_{n}}\right\|_{H S}^{2}-\frac{M N(M-1)(N-1)}{4}\right) \\
& \geq \frac{8}{M N(M+N)}\left(\left(\sum_{n} p_{n}\left\|T_{\psi_{n}}\right\|_{H S}\right)^{2}-\frac{M N(M-1)(N-1)}{4}\right) \\
& \geq \frac{8}{M N(M+N)}\left(\left\|\sum_{n} p_{n} T_{\psi_{n}}\right\|_{H S}^{2}-\frac{M N(M-1)(N-1)}{4}\right) \\
& =\frac{8}{M N(M+N)}\left(\left\|T_{\rho}\right\|_{H S}^{2}-\frac{M N(M-1)(N-1)}{4}\right)
\end{aligned}
$$

where in the first inequality we have used the convexity of the function $f(x)=x^{2}$ and in the second inequality we have used the convexity of $\|\cdot\|_{H S}$ (i.e., the triangle inequality). Equation (26) implies the following separability criterion: $\|T\|_{H S} \leq$ $\sqrt{M N(M-1)(N-1)} / 2$ holds for all separable states. However, this condition is weaker than the CM criterion and can be trivially deduced from it since $\|T\|_{t r} \geq\|T\|_{H S}$. Therefore, (26) will not place new non-trivial bounds where (17) and (18) failed, moreover, the latter equations place non-trivial bounds where the former fails. However, this new bound yields the exact value of the tangle of pure states while the others do not. Thus, although weaker for the detection of entanglement, it will be tighter for the estimation of the tangle for states which are close to pure states. This kind of states are common in experiments where the pure entangled state $\psi$ one aims to prepare is subjected to different types of noise. As a result a slightly mixed state is finally obtained:

$\rho=p|\psi\rangle\langle\psi|+(1-p) \rho_{\text {noise }}$ with $p$ close to 1 . To test the new bound (26), we have considered mixtures in $3 \times 3$ dimensions of arbitrary pure entangled states and white 
noise (i.e. $\rho_{\text {noise }}=I_{9} / 9$ ). We have found that, in general, the bound of (26) can be better than Eqs. (17)-(18) when $p \gtrsim 0.94$.

It is also worth pointing out that using a similar reasoning, it is possible to derive an upper bound for the tangle in terms of $\|\mathbf{r}\|_{2}$ and $\|\mathbf{s}\|_{2}$ if we start from (20) (or its equivalent in terms of $N$ and $\left.\|\mathbf{s}\|_{2}\right)$,

$$
\tau(\rho) \leq 2 \min \left\{\frac{M^{2}-M-2\|\mathbf{r}\|_{2}^{2}}{M^{2}}, \frac{N^{2}-N-2\|\mathbf{s}\|_{2}^{2}}{N^{2}}\right\} .
$$

\subsection{Concurrence and $M N B$ entanglement measure for two-qubit systems}

The MNB measure is an entanglement measure for two-qubit states which is defined as [19]

$$
E(\rho)=\max \left\{\operatorname{tr} \rho^{2}-1+\operatorname{tr} \rho\left(\sigma_{y} \otimes \sigma_{y}\right) \rho^{*}\left(\sigma_{y} \otimes \sigma_{y}\right), 0\right\},
$$

where $\left\{\sigma_{x}, \sigma_{y}, \sigma_{z}\right\}$ denote the standard Pauli matrices (i.e., the generators of $\left.S U(2)\right)$ and $\rho^{*}$ denotes complex conjugation of the density matrix, which is taken, as usual, in the basis of eigenstates of $\sigma_{z}$. Although $E(\rho)=0$ for many entangled states, this measure can be analytically computed and it has been shown to be a rigorous entanglement measure (i.e. non-increasing on average under LOCC) in [20]. In this same paper it is shown that $E(\rho)$ lower bounds $C(\rho)$ for particular sets of states. Based on numerical evidence the authors suggest that this could hold for all states. As an application of our results, we will use the bound (26) to prove this conjecture.

Using equations (5) in [20] and (23) here it is clearly seen that the MNB measure can be written as

$$
E(\rho)=\max \left\{\frac{1}{2}\left(\|T\|_{H S}^{2}-1\right), 0\right\},
$$

which is precisely the bound for the tangle obtained in (26) for two-qubit states. Hence, we have that $\tau(\rho) \geq E(\rho)$. On the other hand, since for two qubits we have that $0 \leq C(\rho) \leq 1$ it holds that $C(\rho) \geq C^{2}(\rho)$. Now, recalling that in this case $\tau(\rho)=C^{2}(\rho)$ we arrive at the desired result: $C(\rho) \geq E(\rho)$. Thus, we see that the MNB entanglement measure is directly related to the $\mathrm{CM}$ and that it lower bounds both the concurrence and tangle.

\section{Conclusions}

The CM criterion provides a general operational sufficient condition for entanglement which, besides its theoretical interest in the theory of entanglement, offers a relatively simple scheme for its detection in experiments. While other important criteria of this type, such as the PPT and CCNR criteria, demand full knowledge of the density operator $\left(M^{2} N^{2}-1\right.$ parameters need to be specified), the CM depends on $\left(M^{2}-1\right)\left(N^{2}-1\right)$ parameters to be determined by measurements of local operators $\left(t_{i j}=M N\left\langle\lambda_{i}^{A} \otimes \lambda_{j}^{B}\right\rangle_{\rho} / 4\right)$ and, hence, the $\mathrm{CM}$ criterion requires in principle less experimental effort. Furthermore, it relies on a measure, $\|T\|_{t r}$, which is left invariant 
under local unitary transformations of the density operator [11] and, therefore, the measurement setups of $A$ and $B$ need not be aligned which, contrary to entanglement tests based on uncertainty relations, also eases its experimental implementation (see [40] for a more detailed discussion).

In this paper we have further confirmed the interest of the CM criterion by providing examples of entangled states which are not detected by the PPT and CCNR criteria whose entanglement is identified by this criterion. Thus, it is clear that the CM criterion together with the previous criteria improves our ability to characterize entanglement, although there remain entangled states unrevealed by the three criteria and the characterization is, therefore, not complete.

Like the PPT and CCNR criteria, the CM criterion can be used to estimate the entanglement content of a state. Here, we have also studied the possibility of quantifying the correlations inherent in the CM by considering a different norm than the one used in the separability criterion. We have found that, although the trace norm is more suitable for the detection of entanglement, the Hilbert-Schmidt norm of the CM is also appropriate for the quantification of entanglement. In fact, it constitutes a rigorous entanglement measure for pure states, given that the concurrence and tangle are monotonously increasing functions of it. Despite that this relation does not hold for mixed states we have shown that it provides a lower bound for the tangle, particularly sharp for states of high purity, that can actually improve the estimations given by the PPT, CCNR and CM criteria. Therefore, the determination of the CM also improves our ability to estimate entanglement measures.

\section{Acknowledgments}

The author thanks O. Gühne for discussions on filter normal forms. Financial support by Universidad Carlos III de Madrid and Comunidad Autónoma de Madrid (Project No. CCG06-UC3M/ESP-0690) and by Dirección General de Investigación (Ministerio de Educación y Ciencia) under Grant No. MTM2006-13000-C03-02 is gratefully acknowledged.

\section{References}

[1] Nielsen M A and Chuang I L 2000 Quantum Computation and Quantum Information (Cambridge University Press)

[2] Gurvits L 2003 Proc. of the 35 Annual ACM Symp. Theory of Computing (San Diego) (New York: ACM Press) p 10

[3] Ioannou L M 2007 Quantum Inf. Comput. 7335

[4] Werner R F 1989 Phys. Rev. A 404277

[5] Peres A 1996 Phys. Rev. Lett. 771413

[6] Horodecki M, Horodecki P and Horodecki R 1996 Phys. Lett. A 2231

[7] Rudolph O 2002 Further results on the cross norm criterion for separability Preprint arXiv:quant-ph/0202121v1

[8] Chen K and Wu L A 2003 Quantum Inf. Comput. 3193 
[9] Lewenstein M, Kraus B, Cirac J I and Horodecki P 2000 Phys. Rev. A 62052310

[10] Hofmann H F and Takeuchi S 2003 Phys. Rev. A 68032103

Gühne O 2004 Phys. Rev. Lett. 92117903

Gühne O, Mechler M, Tóth G and Adam P 2006 Phys. Rev. A 74 010301(R)

[11] de Vicente J I 2007 Quantum Inf. Comput. 7624

[12] Gühne O, Hyllus P, Gittsovich O and Eisert J 2007 Phys. Rev. Lett. 99130504

[13] Plenio M B and Virmani S 2007 Quantum Inf. Comput. 71

[14] Mintert F, Carvalho A R R, Kuś M and Buchleitner A 2005 Phys. Rep. 415207

[15] Chen K, Albeverio S and Fei S M 2005 Phys. Rev. Lett. 95040504

[16] Chen K, Albeverio S and Fei SM 2005 Phys. Rev. Lett. 95210501

Breuer H P 2006 J. Phys. A: Math. Gen. 3911847

Audenaert K M R and Plenio M B 2006 New J. Phys. 8266

Eisert J, Brandão F G S L and Audenaert K M R 2007 New J. Phys. 946

Gühne O, Reimpell M and Werner R F 2007 Phys. Rev. Lett. 98110502

Mintert F 2007 Phys. Rev. A $\mathbf{7 5} 052302$

Zhang C J, Zhang Y S, Zhang S and Guo G C 2007 Phys. Rev. A 76012334

[17] de Vicente J I 2007 Phys. Rev. A 75052320

[18] Datta A, Flammia S T, Shaji A and Caves C M 2007 Phys. Rev. A 75062117

[19] Zhang J, Li C W, Wu J W, Wu R B and Tarn T J 2006 Phys. Rev. A 73022319

[20] Zhang J, Li C W, Tarn T J and Wu J W 2007 Phys. Rev. A 76032306

[21] Horodecki M, Horodecki P and Horodecki R 2006 Open Syst. Inf. Dyn. 13103

[22] Bloch F 1946 Phys. Rev. 70460

[23] Hioe F T and Eberly J H 1981 Phys. Rev. Lett. 47838

[24] Fano U 1983 Rev. Mod. Phys. 55855

[25] Horodecki P 1997 Phys. Lett. A 232333

[26] Horodecki P, Horodecki M and Horodecki R 1999 Phys. Rev. Lett. 821056

Bennett C H, DiVincenzo D P, Mor T, Shor P W, Smolin J A and Terhal B M 1999 Phys. Rev. Lett. 825385

Bruß D and Peres A 2000 Phys. Rev. A 61 030301(R)

[27] DiVincenzo D P, Mor T, Shor P W, Smolin J A and Terhal B M 2003 Comm. Math. Phys. 238379

[28] Breuer H P 2005 J. Phys. A: Math. Gen. 389019

[29] Augusiak R and Stasińska J 2007 Phys. Lett. A 363182

[30] Verstraete F, Dehaene J and De Moor B 2003 Phys. Rev. A 68012103

[31] Leinaas J M, Myrheim J and Ovrum E 2006 Phys. Rev. A 74012313

[32] Verstraete F 2002 Ph. D. Thesis (Katholieke Universiteit Leuven)

[33] DiVincenzo D P and Terhal B M 2001 Proc. of the XIII Int. Cong. on Mathematical Physics (London 2000) (Boston: Int. Press) p 399

[34] Bennett C H, DiVincenzo D P, Smolin J A and Wootters W K, Phys. Rev. A 543824

[35] Wootters W K 1998 Phys. Rev. Lett. 802245

[36] Rungta P, Bužek V, Caves C M, Hillery M and Milburn G J 2001 Phys. Rev. A 64 042315

[37] Rungta P and Caves C M 2003 Phys. Rev. A 67012307

[38] Albeverio S and Fei S M 2001 J. Opt. B: Quantum Semiclass. Opt. 3223

[39] Osborne T J 2005 Phys. Rev. A 72022309

[40] Kothe C and Björk G 2007 Phys. Rev. A 75012336 